Check for updates

Cite this: RSC Adv., 2019, 9, 4314

Received 11th December 2018 Accepted 17th January 2019

DOI: $10.1039 / c 8 r a 10155 h$

rsc.li/rsc-advances

\section{An ultrasonic method for the synthesis, control and optimization of $\mathrm{CdS} / \mathrm{TiO}_{2}$ core-shell nanocomposites}

\begin{abstract}
Sajad Alizadeh, Narges Fallah (D) * and Manochehr Nikazar
In this study, an ultrasonic method was utilized in combination with microemulsion to synthesize $\mathrm{CdS} / \mathrm{TiO}_{2}$ core-shell nanoparticles and control their particle size and ultimately optimize the influential parameters. Moreover, response surface methodology (RSM) was used to optimize the thickness of the shell. Herein, four parameters, i.e. temperature $\left(67-79{ }^{\circ} \mathrm{C}\right.$ ), synthesis retention time (45-105 $\left.\mathrm{min}\right), \mathrm{TiO}_{2}$ : CdS ratio (1.5-7.5) and the power of ultrasound waves (37-53 watt), were optimized to synthesize nanoparticles with an average size of up to $10 \mathrm{~nm}$. A correlation equation was introduced for the size range of 10$90 \mathrm{~nm}$, which was then proven to have excellent predictions. To verify the proposed model, two different sets of combinations were selected to synthesize $10 \mathrm{~nm}$ composites, and consequently, nanocomposites with the sizes of 10.4 and $10.9 \mathrm{~nm}$ were successfully synthesized. The power of ultrasound waves and retention time had the most influence on the size of the particles. Further experiments proved that the optical absorption spectrum of the composite particles was extended to the visible region. Furthermore, the formation of $\mathrm{CdS} / \mathrm{TiO}_{2}$ core-shell nanocomposites was confirmed by different characterization techniques including XRD, TEM, EDAX, UV-vis, FTIR and DLS.
\end{abstract}

\section{Introduction}

The importance of composite nanoparticles, especially core-shell materials, is undeniable not only in the field of chemistry but also in various other fields such as electronics, biomedical optics and catalysis. These materials are highly functional due to their ability to be produced with modified properties. Coating a core with a layer (shell) of different materials can be carried out for numerous purposes such as surface modification, stability, dispersibility, core release control, drug delivery and core consumption. ${ }^{\mathbf{1}}$ Moreover, one of the most interesting features of core-shell composites, which has attracted the attention of scholars from different fields, is that they provide the possibility of combining the advantages of different materials. ${ }^{2,3}$ In the half past century, the advent and advancements in the synthesis of nanoparticles ${ }^{4}$ have led to a significant increase in their popularity; this is mainly due to their physical, chemical and optical features. ${ }^{5}$ Core-shell materials can also help compensate for the disadvantages of individual particles; ${ }^{2}$ one such material, which is widely used and synthesized in many different forms by researchers, is $\mathrm{TiO}_{2}$. $\mathrm{TiO}_{2}$ is a semiconductor that is mostly known in light science. This naturally safe material has fantastic photocatalytic activity in UV light, but has very low photocatalytic activity in visible light. Combining $\mathrm{TiO}_{2}$ with other semiconductors to extend the absorption of $\mathrm{TiO}_{2}$ to the visible region

Chemical Engineering Department, Amirkabir University of Technology, P. O. Box: 15875-4413, Tehran, Iran.E-mail: Nfallah2001@aut.ac.ir is an efficient way to tackle this problem. Several studies have been reported on the synthesis of core-shell and other forms of $\mathrm{TiO}_{2}$-based composites to enhance the photocatalytic and photocorrosion resistance during photodegradation and photocatalytic hydrogen production. ${ }^{6-10}$ The major problem in the synthesis of core-shell materials is the difficulty of coating the materials, especially inorganic components, on a small core. This is mainly due to the lack of proper coating methods as well as weak surface forces existing under normal conditions. The results achieved under normal conditions using conventional methods for the synthesis of core-shell particles, especially in the process of coupling semiconductors, may not be efficient since a quite long retention time (about one day or more) and high temperature (200-400 ${ }^{\circ} \mathrm{C}$ ) are required for sufficient interfacial adhesion and crystallinity of both components. ${ }^{11-15}$ In this regard, one of the promising approaches that can overcome the aforementioned problems is the sonochemical method, in which ultrasound waves are utilized for the synthesis of different types of nanoparticles with a narrow size distribution under mild conditions. ${ }^{16-18}$ During irradiation of liquids with ultrasound, a phenomenon called cavitation takes place. The produced cavity serves as a mean to concentrate the diffused sound energy. In any acoustic field, once a microbubble experiences rapid growth, it can no longer efficiently absorb energy, and thus, the liquid will rush in, and the cavity will eventually implode. ${ }^{19,20}$ Once these grown bubbles collapse and implode, they act as a hotspot and generate energy, which can increase the local pressure and temperature to $500 \mathrm{~atm}$ and $5000{ }^{\circ} \mathrm{C}$, respectively. Then, the 
liquid will cool down at the rate of $10^{9} \mathrm{~K} \mathrm{~s}^{-1} \cdot{ }^{20}$ These collapsing bubbles create a turbulence synthesis environment, an unusual mechanism for chemical reactions which need high level of energy to occur. The turbulent media and the consequent high velocity field can help with easier deposition of organic or inorganic materials on a small core as compared to the case of conventional methods. Due to these features, the use of ultrasonic waves has become a promising method for the synthesis of a wide range of materials ranging from inorganic/polymeric coreshell materials as a system modifier ${ }^{21}$ to inorganic/inorganic core-shell catalysts; ${ }^{2}$ although studies on the formation of CdS/ $\mathrm{TiO}_{2}$ core-shell composites using ultrasound waves are quite rare, some studies on the formation of $\mathrm{TiO}_{2} / \mathrm{CdS}$ core-shell materials with a hexagonal phase and size in the range of 25$30 \mathrm{~nm}$ under a multi-bubble sonoluminescence (MBSL) condition are available. ${ }^{22}$ Note that not only the size of the particles obtained in this process is larger but also the complexity of the sonoluminescence reactor is a major drawback. In addition, synthesis of 0- and 1-dimensional mesoporous core-shell CdS/ $\mathrm{TiO}_{2}$ with an average size of $20 \mathrm{~nm}$ through a sol-gel method was investigated. ${ }^{23}$ However, the sol-gel method and solvothermalassisted synthesis, which have been used to prepare these CdS/ $\mathrm{TiO}_{2}$ core-shells, are time-consuming; ${ }^{23}$ one of the most successful approaches to synthesize these particles is the sonochemical method, in which the complexity of the sonoluminescence reactor and the long duration of solvothermal synthesis are completely eliminated. ${ }^{2}$ Thus, it seems that the ultrasound approach would be a more promising way for the synthesis or at least for coating the nanoparticles. Use of ultrasonic waves with an experimental design approach, such as response surface methodology, can efficiently increase the performance of the synthesis; this is due to the possibility for the optimization of response, extrapolation of the results to unexamined data points and an accurate estimation of the extrapolated points. Among the RSM methods, the central composite design (CCD), which is a $2^{\mathrm{k}}$ factorial design with two variables and $n_{\mathrm{c}}$ central point, is very famous. This method of design usually provides the best answers due to the reasonable distribution of points across the desired area. ${ }^{24}$ The aim of the present study was to synthesize CdS nanoparticles and then coat them with a $\mathrm{TiO}_{2}$ layer using an ultrasonic method. The targeted size of the final nanocomposite was $10 \mathrm{~nm}$; thus, the influential parameters (i.e. temperature, time, $\mathrm{TiO}_{2}$ : CdS ratio and power of sound beam) controlling the thickness of the shell were optimized accordingly using the RSM method, and a model was obtained. The model could also be used to synthesize $\mathrm{CdS} / \mathrm{TiO}_{2}$ with different sizes. The other aspect of this study was to investigate the importance and impact of these parameters on the final composite size.

\section{Materials and methods}

\subsection{Materials}

Ethylenediamine, titanium tetra-isopropoxide (TTIP), $\mathrm{CS}_{2}$ and $\mathrm{CdCl}_{2} \cdot \mathrm{H}_{2} \mathrm{O}$ were supplied by Merck Company and used without any further purification. Distilled water was used for all the syntheses and sample preparation steps.

\subsection{Methods}

2.2.1. Synthesis of the CdS nanoparticles. Typically, $1 \mathrm{~mL}$ of ethylenediamine was added to $50 \mathrm{~mL}$ water in a beaker at room temperature, and then, $0.3 \mathrm{~mL}$ of $\mathrm{CS}_{2}$ was added to complete the oil phase, which was irradiated for $20 \mathrm{~min}$ using ultrasound waves (TOMY, UD 201, acoustic power $45 \mathrm{~W}$ with digital water bath temperature controller) at $40{ }^{\circ} \mathrm{C}$ until the solution became clear. Then, an aqueous solution of hydrated cadmium chloride $(0.3 \mathrm{~g}$ dissolved in $15 \mathrm{~mL}$ of distilled water) was added to the beaker as an aqueous phase, and the entire solution was sonicated for about 15 min without changing the temperature. After the mixing process, the temperature was increased to about $75{ }^{\circ} \mathrm{C}$ for $10 \mathrm{~min}$ for the nucleation of the CdS nanoparticles, and then, it was kept constant for another 30 minutes. ${ }^{25}$ The CdS nanoparticles were obtained via centrifugation, and then, the precipitate was separated, washed with distilled water and ethanol, and dried at room temperature. Finally, $0.05 \mathrm{~g}$ of dried CdS particles was added to $20 \mathrm{~mL}$ distilled water followed by sonication for 5 minutes to achieve uniform dispersion. Then, $5 \mathrm{~mL}$ of this solution was characterized using dynamic light scattering to measure the particle size and size distribution.

2.2.2. Synthesis of core-shell nanoparticles. To coat the CdS nanoparticles with $\mathrm{TiO}_{2}$, titanium isopropoxide (TTIP) was added drop-wise to the solution in an ultrasonic medium. The final product was separated by centrifugation (5000 rpm for 20 minutes), washed with absolute ethanol and distilled water, and dried in an oven at $70{ }^{\circ} \mathrm{C}$ for 1 hour. Further, the parameters, i.e., time, temperature, $\mathrm{TiO}_{2}$ : CdS ratio and power of the sonic beams, that affect the thickness of the shell were optimized according to the statistical experimental design. The goal of this optimization was to achieve core-shell structures with desirable size. $^{26}$

\subsection{Characterization of the nanocomposite}

The structure and morphology of the products were investigated using X-ray diffraction (Bruker D8 Advanced), energy dispersive X-ray spectroscopy (Philips XL 30S FEG), transmission electron microscopy (Philips, CM120 Bio-TWIN), dynamic light scattering (HORIBA - LB550), and FTIR spectroscopy (Perkin Elmer - Spectrum RX I); moreover, ultraviolet-visible spectroscopy (Perkin Elmer, Lambda 850) was employed to study the products from a photocatalytic point of view.

\subsection{Experiment design}

Analysis of experimental data and the suggested experiments were carried out by Design-expert ${ }^{\circledR}$ version 7.0. An RSM based on the central composite design (CCD) was applied to determine the number and conditions of the experiments needed to develop a model. Each numerical factor varied at five levels. Moreover, thirty experiments were suggested by the CCD method for the optimization of the influential parameters. In the suggested sets of experiments, four independent variables (time, temperature, molar ratio of $\mathrm{TiO}_{2}$ to $\mathrm{CdS}$ and power of sonic beams) were used in the RSM. Each variable was found to 


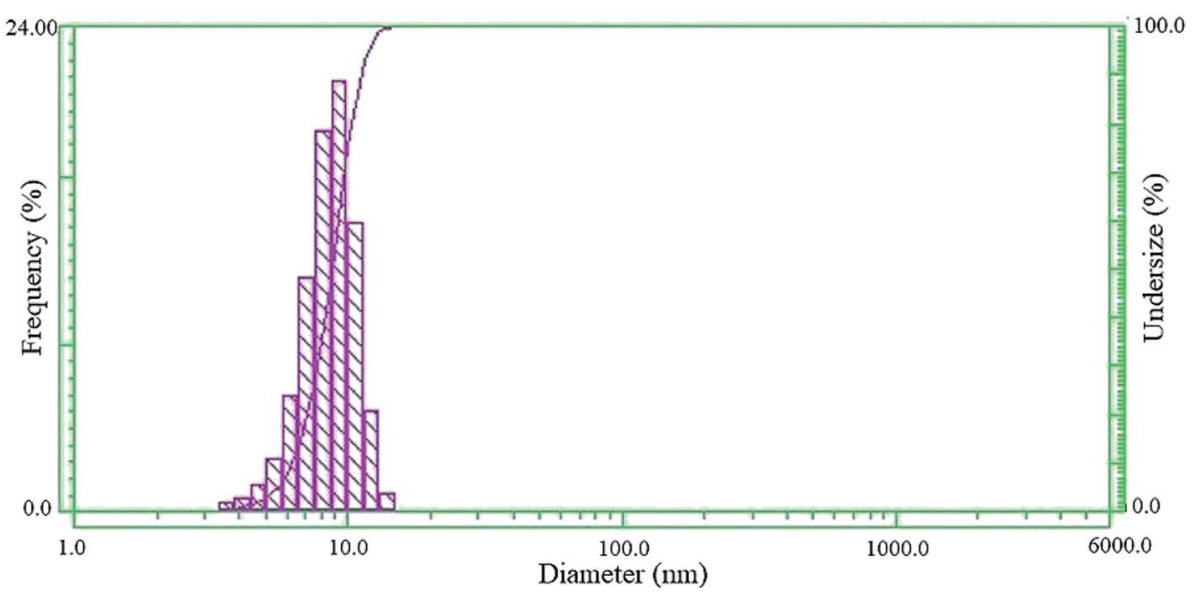

Fig. 1 DLS pattern of the CdS nanoparticles.

be within the range suggested in previous studies., ${ }^{2,11,13}$ In addition, to improve the synthesis model, a level higher and lower than the suggested levels were selected and investigated. Table 1 shows the parameters used in the RSM design using the CCD method. The units and the range of change for each parameter are presented. (The range of change appears in terms of \pm alpha.)

\section{Results and discussion}

\subsection{Core size analysis}

Fig. 1 shows the DLS pattern of the synthesized CdS core. The pattern indicates a narrow size distribution of the synthesized CdS nanoparticles with an average size of $8.7 \mathrm{~nm}$. As shown in the pattern, the particle size varied between 3.5 and $10.5 \mathrm{~nm}$, and the most intense peak (about 26\%) occurred at $9 \mathrm{~nm}$.

\subsection{Statistical analysis}

The response used in the model was the size of the synthesized nanocomposite in each experiment. The size of each composite was measured using the DLS method. Table 2 represents the influential parameters, the suggested combinations and the output results. (*Indicates center point experiments.)

To assess the statistical and mathematical adequacy of the proposed model, a plot comparing the predicted response values of the model with the experimental data was generated (Fig. 2), which showed a good agreement between the values predicted by the model and the observed values. Based on the

Table 1 Core-shell and CCD parameters ${ }^{a}$

\begin{tabular}{lllllll}
\hline & & & -1 & +1 & - & + \\
& Name & Unit & Level & Level & Alpha & Alpha \\
\hline $\mathrm{A}$ & Temperature & ${ }^{\circ} \mathrm{C}$ & 70 & 76 & 67 & 79 \\
$\mathrm{~B}$ & $\mathrm{Time}_{\mathrm{C}}$ & $\mathrm{min}$ & 60 & 90 & 45 & 105 \\
$\mathrm{C}$ & $\mathrm{TiO}_{2} / \mathrm{CdS}$ & Molar ratio & 3 & 6 & 1.5 & 7.5 \\
$\mathrm{D}$ & Wave power & Watt & 41 & 49 & 37 & 53
\end{tabular}

${ }^{a}$ Alpha: 2, replicates of factorial points: 1, replicates of axial points: 1 , center point: 6 . experimental data listed in Table 2, an empirical equation was derived as follows:

$$
\begin{aligned}
S^{-0.14}= & -0.72235-0.015873 \times \mathrm{Te}-3.24399 \times 10^{-3} \times \mathrm{Ti}- \\
& 0.035904 \times \mathrm{T}: \mathrm{C}+0.090528 \times \mathrm{WP}+3.66045 \times 10^{-4} \\
\times & \mathrm{Te} \times \mathrm{WP}+4.01792 \times 10^{-4} \times \mathrm{Ti} \times \mathrm{Tc}- \\
& 1.21566 \times 10^{-3} \times \mathrm{WP}^{2}
\end{aligned}
$$

where $S, \mathrm{Te}, \mathrm{Ti}, \mathrm{T}: \mathrm{C}$ and WP represent the core-shell size, temperature, time, $\mathrm{TiO}_{2}$ : CdS ratio and the power of waves, respectively.

$$
\begin{aligned}
S^{-0.14}= & 0.66+1.793 \times 10^{-3} \times A-0.022 \times B-8.654 \times 10^{-3} \\
& \times C+0.031 \times D+4.393 \times 10^{-3} \times A \times D+9.040 \\
& \times 10^{-3} \times B \times C-0.019 \times D^{2}
\end{aligned}
$$

where $S, A, B, C$ and $D$ represent the core-shell size, temperature, time, $\mathrm{TiO}_{2}$ : CdS ratio and the power of waves, respectively. Eqn (2) is the coded form of eqn (1). In this equation, the positive values indicate a favourable effect of the parameter on the response, whereas the negative values represent an antagonistic effect on the response (an inverse relationship); moreover, the magnitude of these numbers shows the extent of the impact of each factor. ${ }^{27,28}$ As eqn (2) indicates, the positive sign for $A$ and $D$ allow the particles to have a smaller size. In addition, the magnitude of coefficient indicates that the impact of $D$ is far more than that of $A$. On the other hand, the negative signs for $B$ and $C$ indicate an antagonistic effect i.e. the higher these parameters, the greater the particle growth. In this case, the retention time has more impact on the particle size than the precursor ratio. Statistical analysis (ANOVA) proved that the model was significant as the $F$ value for the model was 24.95 and the corresponding $p$ value was $<0.0001$. This would mean that there was only a $0.01 \%$ chance that this $F$-value for the model could occur due to noise. The lack of fit $F$ value of 0.07 was not considered significant as the $p$ value was 1.000 . For a model to have a successful prediction, the lack of fit should not be significant. Moreover, the adjusted $R$-squared value is very close to the corresponding $R$-squared value; this further confirms the adaptability of this model. ${ }^{29}$ The predicted $R$-squared of 0.8909 
Table 2 Output data for the suggested experiments ${ }^{a}$

\begin{tabular}{|c|c|c|c|c|c|c|c|c|c|c|c|}
\hline Run & $\begin{array}{l}\text { Temperature } \\
\left({ }^{\circ} \mathrm{C}\right)\end{array}$ & Time (min) & Power (watt) & $\begin{array}{l}\text { Ratio } \\
\text { (molar ratio) }\end{array}$ & Size (nm) & Run & $\begin{array}{l}\text { Temperature } \\
\left({ }^{\circ} \mathrm{C}\right)\end{array}$ & Time (min) & Power (watt) & $\begin{array}{l}\text { Ratio } \\
\text { (molar ratio) }\end{array}$ & Size (nm) \\
\hline 1 & 73.00 & 75.00 & 45.00 & 4.50 & $16.63^{*}$ & 16 & 73.00 & 75.00 & 45.00 & 4.50 & $17.42 *$ \\
\hline 3 & 73.00 & 75.00 & 45.00 & 4.50 & $17.24^{*}$ & 18 & 73.00 & 75.00 & 45.00 & 4.50 & $16.41^{*}$ \\
\hline 4 & 70.00 & 90.00 & 49.00 & 6.00 & 22.7 & 19 & 76.00 & 60.00 & 41.00 & 6.00 & 33.25 \\
\hline 5 & 70.00 & 60.00 & 41.00 & 6.00 & 29.41 & 20 & 76.00 & 60.00 & 49.00 & 3.00 & 10.12 \\
\hline 8 & 73.00 & 75.00 & 53.00 & 4.50 & 20.13 & 23 & 73.00 & 75.00 & 45.00 & 4.50 & $17.05^{*}$ \\
\hline 9 & 70.00 & 60.00 & 41.00 & 3.00 & 23.65 & 24 & 73.00 & 45.00 & 45.00 & 4.50 & 15.19 \\
\hline 10 & 76.00 & 60.00 & 41.00 & 3.00 & 24.62 & 25 & 67.00 & 75.00 & 45.00 & 4.50 & 19.19 \\
\hline 11 & 70.00 & 90.00 & 41.00 & 3.00 & 42.12 & 26 & 76.00 & 90.00 & 41.00 & 3.00 & 37.8 \\
\hline 12 & 70.00 & 90.00 & 49.00 & 3.00 & 23.85 & 27 & 73.00 & 75.00 & 45.00 & 4.50 & $15.94^{*}$ \\
\hline 13 & 76.00 & 90.00 & 41.00 & 6.00 & 49.52 & 28 & 76.00 & 60.00 & 49.00 & 6.00 & 17.74 \\
\hline
\end{tabular}

${ }^{a}$ Statistical analysis of the output data obtained from the experiments was performed by Design Expert® version 7.0.0.

is in reasonable agreement with the adjusted $R$-squared of 0.9326. Adequate precision is determined by the signal to noise ratio. A ratio greater than 4 is desirable, and the obtained ratio has been found to be 22.848 , which indicates an adequate signal and also proves that this model can be used to navigate the design space.

\subsection{Effect of the factors affecting the particle size}

The perturbation diagram, as shown in Fig. 3, is useful for predicting the impact of different parameters on the particle size of the nanocomposite.
Line (A) from left to right indicates an increase in temperature, which leads to a reduction in the size of the synthesized particles. Temperature shows the smallest effect on the particle size. By increasing the time of synthesis, the residual $\mathrm{TiO}_{2}$ particles are allowed to hit and join the formed shell; this increases the shell thickness, and consequently, the size of the core-shell is increased, as indicated by Line (B). Moreover, an increase in the ratio of the precursors led to growth in the size of the synthesized particles. Line (C) indicates the increase in $\mathrm{TiO}_{2}$ to $\mathrm{CdS}$ precursor ratio. As the amount of $\mathrm{TiO}_{2}$ particles in the mixture increased, the probability of formation of a denser and

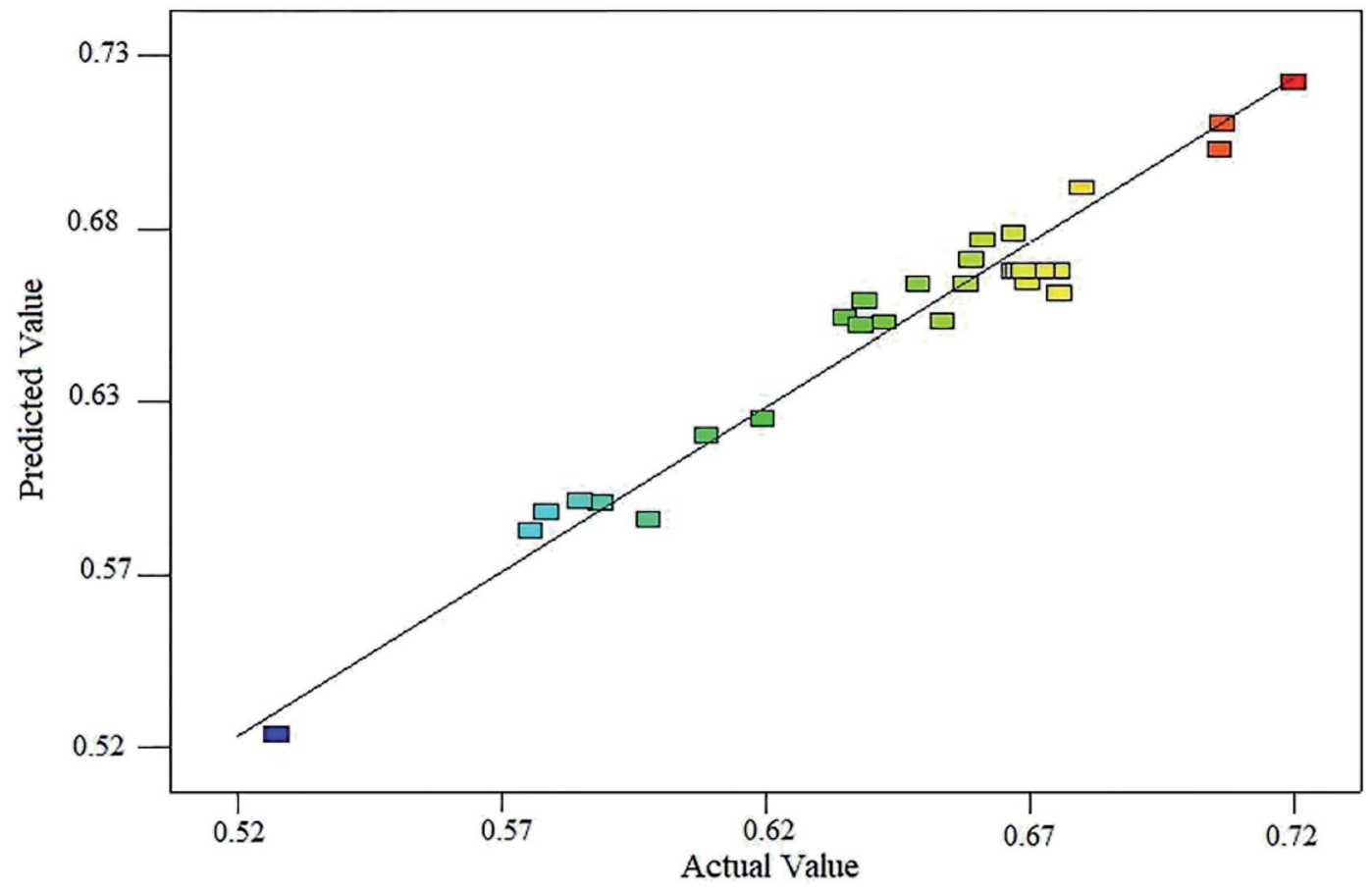

Fig. 2 Relationship between the predicted and experimental sizes of the synthesized nanocomposite. 


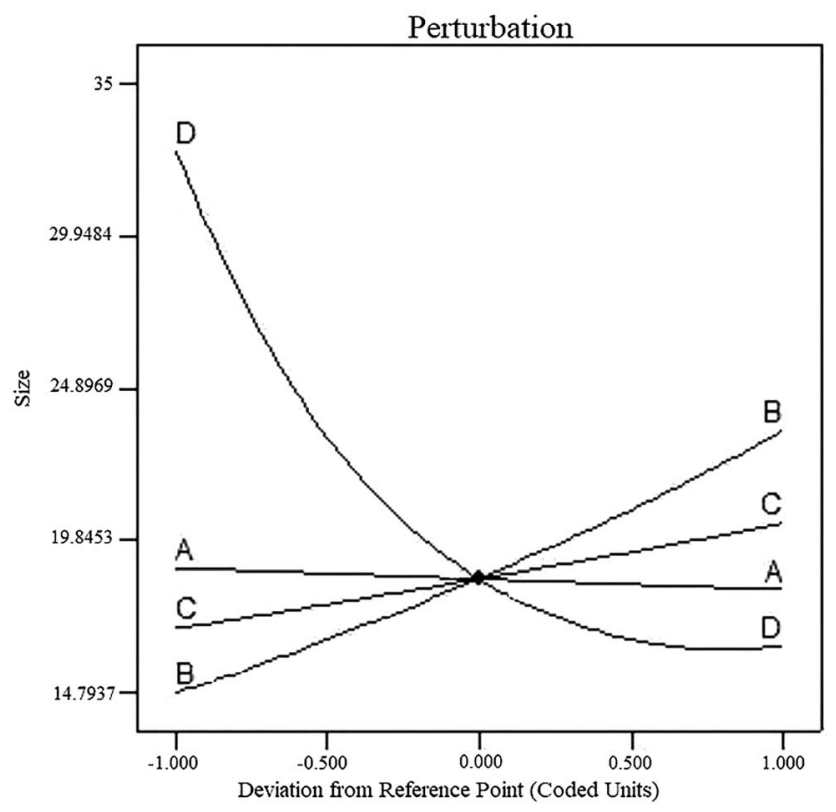

Fig. 3 Perturbation diagram of the model.

thicker shell also increased due to higher amounts of the $\mathrm{TiO}_{2}$ precursor (TTIP) than those of the CdS precursors $\left(\mathrm{CS}_{2}\right.$ and $\mathrm{CdCl}_{2} \cdot \mathrm{H}_{2} \mathrm{O}$ ). This phenomenon was almost similar to that of retention time, but the influence of retention time was far more significant than that of the ratio of components. As the power of the ultrasound waves increases, the level of turbulence in the system will also increase, and the particles will collide each other with higher momentum and the chances for the formation of agglomerate particles will definitely diminish. This phenomenon is shown by curve (D), and as it can be seen, by increasing the power of the ultrasound waves, the particle size of the final product can be decreased. The interactions between parameters were studied using the contour plots shown in Fig. 4. As the perturbation diagram indicates, temperature has the least influence on the size as compared to the other factors. In fact, if one of the other factors remained constant, a change in temperature could not alter the size of particles. This behavior can be observed in Fig. 4a, $\mathrm{d}$ and $\mathrm{f}$. The most important interaction was between the synthesis retention time and the power of sonic waves, which acted in opposite directions. Upon increasing the power of beams, the size of the particles reduced, and an increase in retention time led to an increase in the particle size. Note that the synthesis of fine particles requires adequate power of ultrasound. However, the contours in Fig. 4c indicate that after a specific retention time, any further increase in the power of sonic waves is ineffective since it can no longer overcome the influence of retention time, and thus, the particle size will increase. The interaction between the sonic waves and the precursor ratio can be regarded the same as the interaction between the synthesis retention time and the power of sonic waves. Their influence is fairly smoother as compared to that of the retention time; however, it cannot be ignored. For example, Fig. 4e indicates that at the center points of temperature and retention time, $\mathrm{TiO}_{2}: \mathrm{CdS}$ ratios greater than 5.3 cannot lead to particles smaller than $17 \mathrm{~nm}$. The last interaction studied was between the retention time and the precursor ratio, and since both these parameters act in favor of the increase in the particle size, their interaction led to a constructive interference, and ultimately, the final size of the particles increased.

\subsection{Optimization of core-shell}

As the goal of this study was to achieve a size of $10 \mathrm{~nm}$, the model was optimized accordingly. Thus, the size of the particle was intentionally maintained constant at $10 \mathrm{~nm}$, whereas the
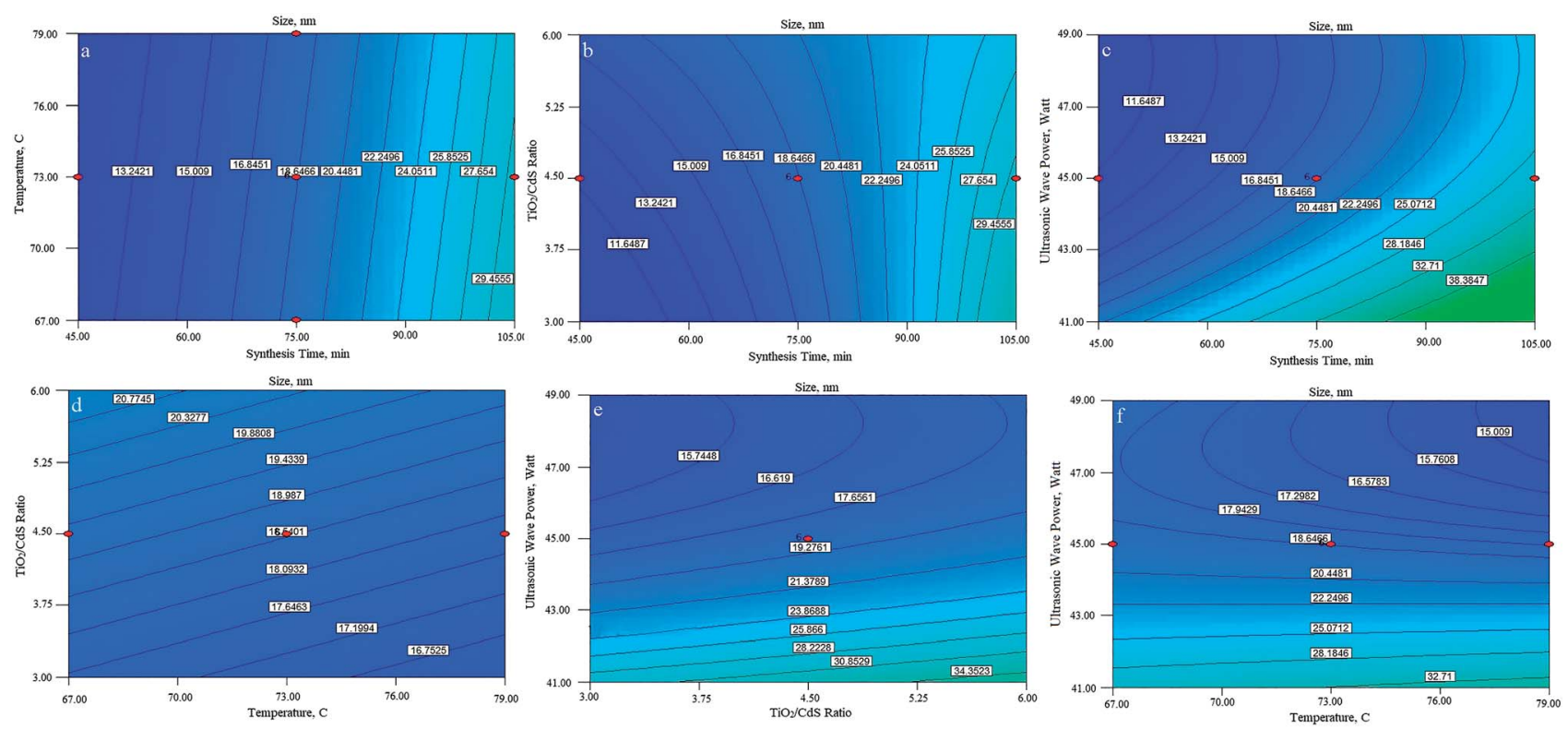

Fig. 4 Contour plots of the different parameters affecting the particle size. 
Table 3 Optimization factors, goals, limits and importance of each parameter

\begin{tabular}{|c|c|c|c|c|c|c|}
\hline Name & Goal & Lower limit & Upper limit & Lower weight & Upper weight & Importance \\
\hline Temperature & Is in range & 67 & 79 & 0.231101 & 0.301407 & 5 \\
\hline Time & Is in range & 45 & 105 & 1 & 0.265826 & 3 \\
\hline Wave power & Is in range & 37 & 51 & 1 & 1 & 3 \\
\hline Size & Is $10 \mathrm{~nm}$ & 11.57 & 91.62 & 0.1 & 0.265826 & 5 \\
\hline
\end{tabular}

other parameters were changed within their operational range except for the $\mathrm{TiO}_{2}$ : CdS ratio. This ratio was purposely minimized due to financial consideration and the high cost of the initial precursors. Table 3 shows the optimization parameters, their goal and importance. As shown, the optimization process suggested the relationships between parameters for achieving a $10 \mathrm{~nm}$ composite particle, and the ratio between precursors is minimal.

Some of these relations are shown in Table 4. To assess the model, two different combinations with different amounts of $\mathrm{TiO}_{2}$ : CdS precursors were selected from the table: 2.97 and 4.87 for composite A and composite B, respectively.

\subsection{Characterization of the synthesized core-shell composite}

Fig. 5 shows the DLS patterns representing the size distribution of the $\mathrm{CdS} / \mathrm{TiO}_{2}$ particles. As the plots shows, particles of both composites have narrow distribution with the average sizes of 10.4 and $10.9 \mathrm{~nm}$. The actual data shows only 0.4 and $0.9 \mathrm{~nm}$ difference as compared to the model prediction.

Fig. 6 shows the XRD patterns of composite A, composite B, $\mathrm{TiO}_{2}$ and $\mathrm{CdS}$ (individual component patterns are obtained from the HighScore Plus database version 2013). The XRD patterns reveal that the $\mathrm{TiO}_{2}$ nanoparticles are in anatase phase with intense peaks at $2 \theta=24.9^{\circ}, 26.5^{\circ}, 28.2^{\circ}, 36.8^{\circ}, 44^{\circ}$, and $48.1^{\circ}$, and the CdS nanoparticles have intense peaks at $2 \theta=$ $27.5^{\circ}, 36.1^{\circ}, 54.5^{\circ}$, and $69.1^{\circ}$. The relatively narrow XRD peaks of both composites show that the particle sizes are in nano scale, and the thickness of the $\mathrm{TiO}_{2}$ shell formed on CdS nanoparticles is less. A comparison between the X-ray diffraction patterns of composites A and B revealed that the lower peak breadth of composite A represented a smaller size of the product, and consequently, a shell containing less amount of deposited $\mathrm{TiO}_{2}$ was obtained on the CdS core.

Chemical composition analysis by the EDAX method showed moderate to high purity for each component in both the CA and $\mathrm{CB}$ composites. The analysis also revealed that the atomic percentages of $\mathrm{Cd}, \mathrm{S}$ and $\mathrm{Ti}$ were $20.62,21.93$ and 57.44 in the composite A and 13.85, 15.92 and 70.21 in the composite B, respectively. This means that the atomic ratios between $\mathrm{Cd}$ and $S$ are almost 1:1 in both composites, which indicates that about $21.2 \%$ and $14.9 \%$ of composite A and B have been made by the CdS particles, respectively. Consequently, the ratio of $\mathrm{TiO}_{2}$ : Cds in composites A and B would be $2.71: 1$ and $4.71: 1$, respectively (2.97: 1 and $4.87: 1$, as listed in Table 2). Fig. 7 shows the EDAX patterns of both composites A and B.

The efficiency of encapsulation of both composites, actual size of individual composite and shapes of the cores and shells

Table 4 A few combinations of the synthesis parameters for synthesizing a $10 \mathrm{~nm}$ composite

\begin{tabular}{|c|c|c|c|c|c|c|c|}
\hline Solutions number & $\begin{array}{l}\text { Temperature } \\
\left({ }^{\circ} \mathrm{C}\right)\end{array}$ & Time (min) & $\begin{array}{l}\mathrm{TiO}_{2}: \text { CdS } \\
\text { (molar ratio) }\end{array}$ & $\begin{array}{l}\text { Wave power } \\
\text { (watt) }\end{array}$ & Size $(\mathrm{nm})$ & Desirability & Result \\
\hline 1 & 70.26 & 46.92 & 3.73 & 48.82 & 10.001 & 0.961 & \\
\hline 2 & 67.72 & 49.66 & 2.97 & 49.37 & 10 & 0.951 & Selected (CA) \\
\hline 3 & 77.46 & 64.41 & 1.94 & 47.68 & 10.001 & 0.945 & \\
\hline 4 & 72.13 & 56.07 & 1.99 & 45.00 & 10.001 & 0.918 & \\
\hline 5 & 78.95 & 45.32 & 4.87 & 49.15 & 9.999 & 0.914 & Selected (CB) \\
\hline
\end{tabular}
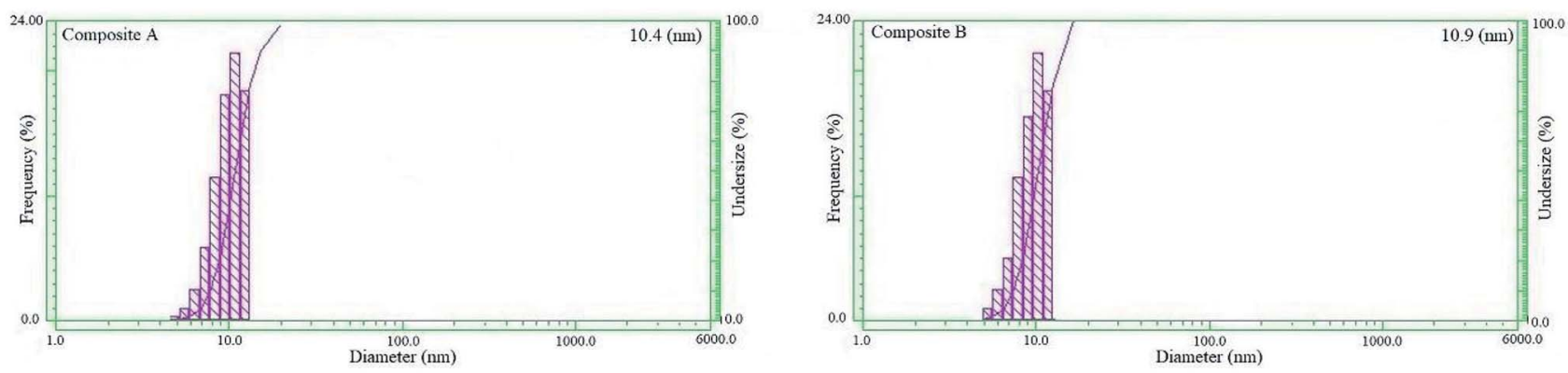

Fig. 5 DLS analysis of the nanocomposites. 

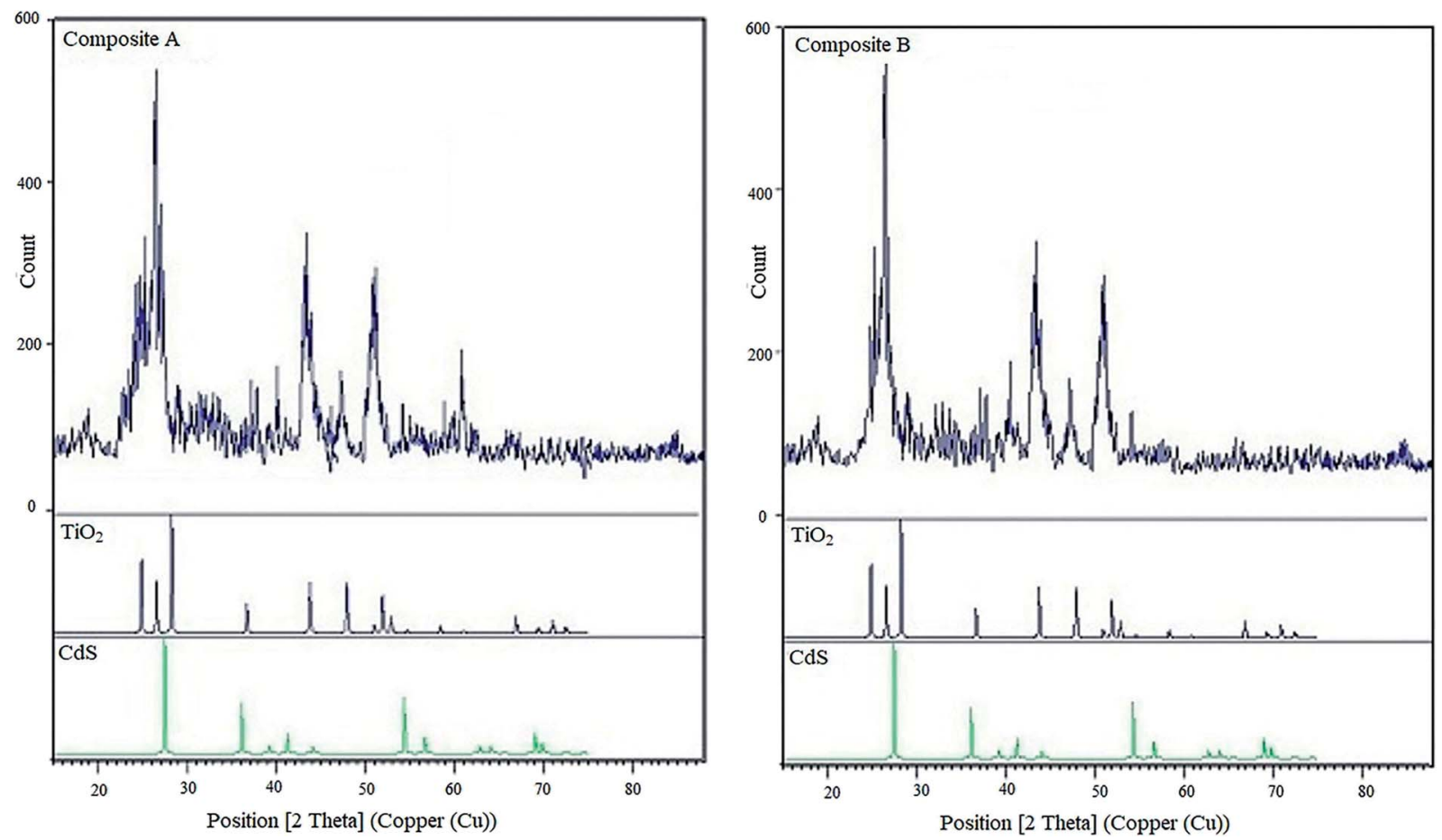

Fig. 6 XRD patterns of composite $A$, composite $\mathrm{B}$ and the individual patterns of $\mathrm{TiO}_{2}$ and $\mathrm{CdS}$.

were investigated by TEM. The images clearly demonstrated that a nano-sized layer of titanium oxide $(0.9$ and $1 \mathrm{~nm}$ respectively for composite A \& B) fully enclosed the $9 \mathrm{~nm}$ CdS core. The spherical shape of the cores is speculated to have arisen due to the high-velocity synthesis field. Cavitation phenomena and the intense change in local temperature and pressure lead to the development of a high velocity field. In this field, particles collide each other with a great momentum. This great momentum leads the particles to reach their most stable shape in a three-dimensional medium. In a fluid medium, sphere is the most stable shape, and our TEM images prove this fact. Fig. 8 shows the TEM images of both the composite A \& the composite B particles.
Fig. 9 illustrates the FTIR spectra of the composites A and B over $500-2500 \mathrm{~cm}^{-1}$. The reference charts indicate that the peak in the region $400-600 \mathrm{~cm}^{-1}$ is due to the transverse optical active vibration of the Ti-O bonds, whereas the peak in the region $700-950 \mathrm{~cm}^{-1}$ is due to the longitudinal optical (LO) vibrational mode. ${ }^{30}$ The sharp peak at $1400 \mathrm{~cm}^{-1}$ can be recognized to be due to the lattice vibrations of $\mathrm{TiO}_{2} \cdot{ }^{31} \mathrm{In}$ addition, a binding vibration associated with $\mathrm{Ti}-\mathrm{OH}$ can be seen as an absorption band at $1627 \mathrm{~cm}^{-1} \cdot{ }^{30}$ For CdS, the reference charts indicate peaks at 619 and $659 \mathrm{~cm}^{-1}$ due to Cd-S stretching. ${ }^{32}$ Moreover, the peaks at 1391 and $1630 \mathrm{~cm}^{-1}$ indicate the lattice vibrations of CdS. ${ }^{30}$ As shown in Fig. 9, the peaks in the $500-700 \mathrm{~cm}^{-1}$ region indicate an overlap between $\mathrm{TiO}_{2}$
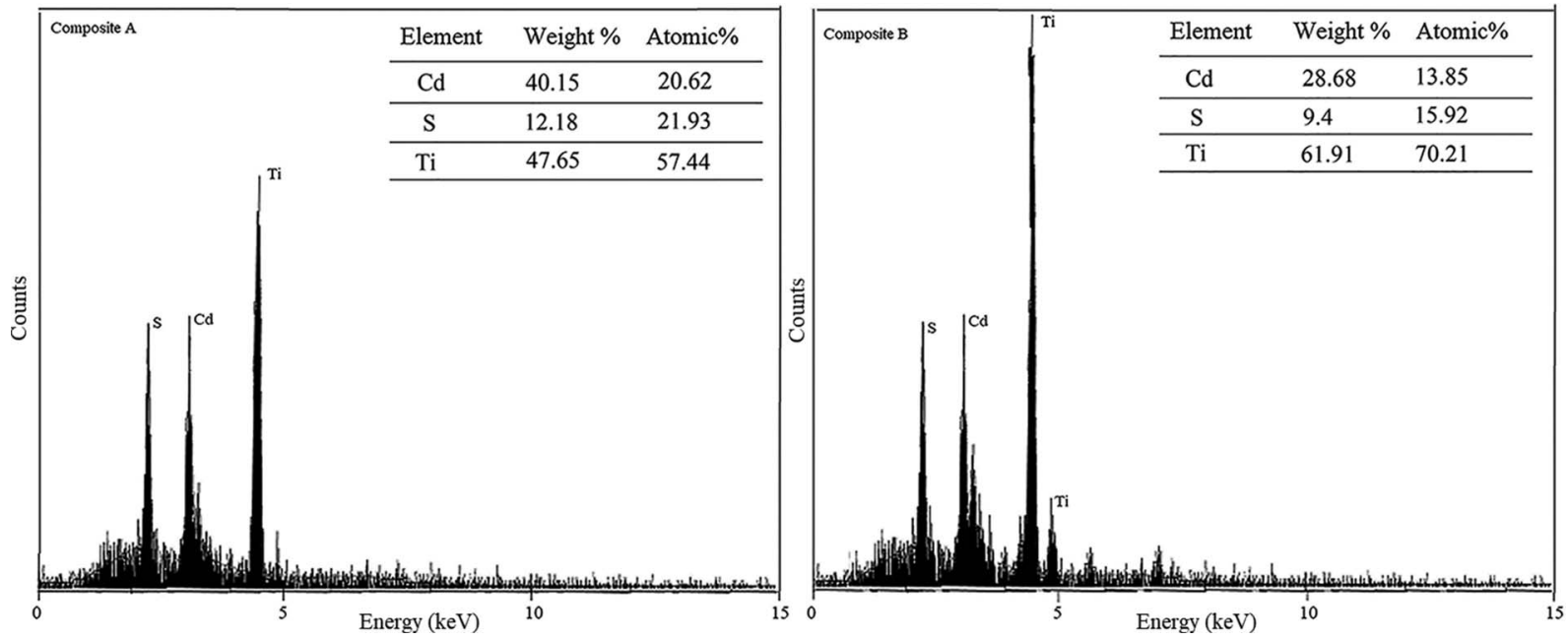

Fig. 7 EDAX patterns of composites $A$ and $B$. 


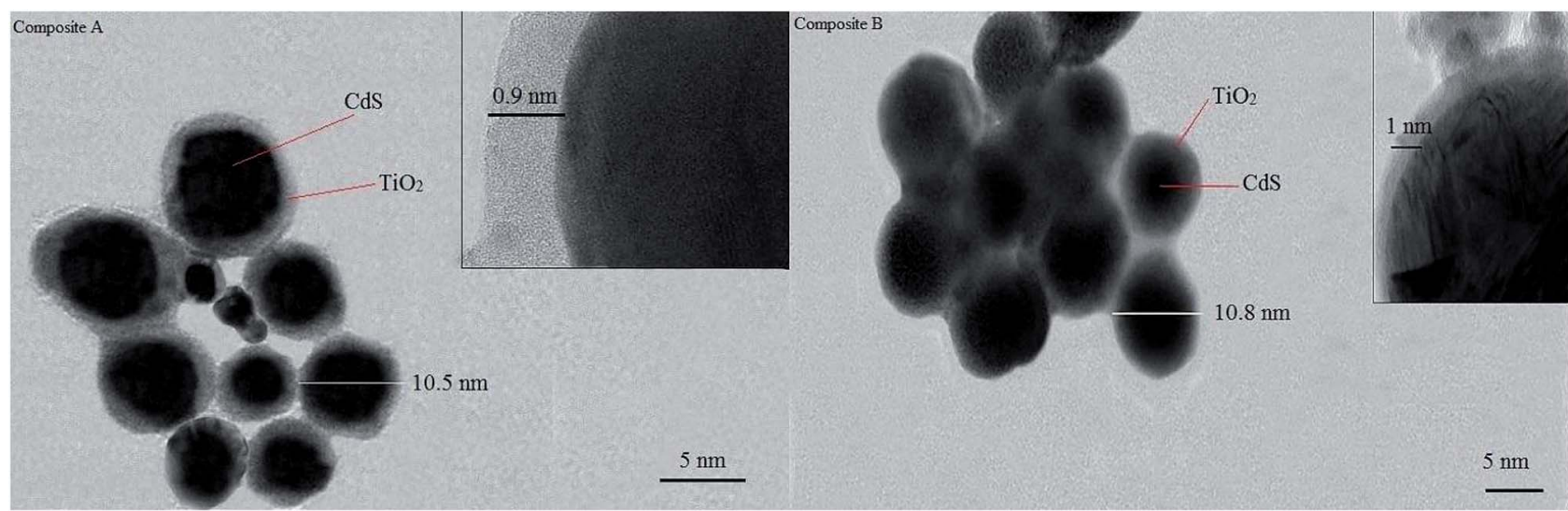

Fig. 8 TEM images of the nanocomposites $\mathrm{A}$ and $\mathrm{B}$.

and CdS, and the peaks at 1380, 1410 and $1650 \mathrm{~cm}^{-1}$ clearly reveal that the CdS molecules are attached to $\mathrm{TiO}_{2}$.

In photocatalysis research, UV-vis absorption spectroscopy is one of the most important characteristic tests to monitor the band gap shifts. Fig. 10 represents the UV-vis absorption spectra of both composites as well as pure components. As the spectra indicates, the absorption of composite B is in a lower wavelength region as compared to that of composite A. This phenomenon is due to the higher ratio of $\mathrm{TiO}_{2}$ : CdS and the thicker shell of composite $\mathrm{B}$. The band gap plots for these components are illustrated in Fig. 11. Pure $\mathrm{TiO}_{2}$ and $\mathrm{CdS}$ nanoparticles show the band gap energies of 3.39 and $2.45 \mathrm{eV}$, whereas the composite A \& composite B show the band gap energies of 2.82 and $2.95 \mathrm{eV}$, respectively. Participation of $\mathrm{TiO}_{2}$ and CdS in the core-shell system has led to a red shift in the absorption spectra of pure $\mathrm{TiO}_{2}$, and the optical absorption has extended to the visible region as compared to that of the pure $\mathrm{TiO}_{2}$ particles. Red shift of the spectrum is a typical characteristic of core-shell nanocrystals with higher band gap shells due to reduction in the surface defects of the core. ${ }^{33}$ The $E_{\mathrm{g}}$ or band gap of samples has been determined by plotting the following equation (eqn (3) $)^{34}$ and extrapolating the linear portion, which intercepts the energy axis $h \nu$.

$$
\alpha h \nu=A\left(h \nu-E_{\mathrm{g}}\right)^{\frac{n}{2}}
$$

where $\alpha$ is the absorption coefficient, $h$ is the Planck's constant, $\nu$ is the light frequency, $n$ is a proportionality constant, and $A$ and $E_{\mathrm{g}}$ are the constant and the band gap of the nanoparticle, respectively. The exponent ' $n$ ' is determined by the transition of semiconductor, for example, a direct transition for zinc ferrite and cadmium sulfide $(n=1),{ }^{2,34,35}$ and an indirect transition for $\mathrm{TiO}_{2}(n=4) \cdot{ }^{34}$ As shown in Fig. 11, the indirect measurement of optical transition shows a reduction in the band gap for both composites with respect to pure $\mathrm{TiO}_{2}$. Moreover, the composite A shows more reduction in energy due to a lower ratio of $\mathrm{TiO}_{2} / \mathrm{CdS}$.

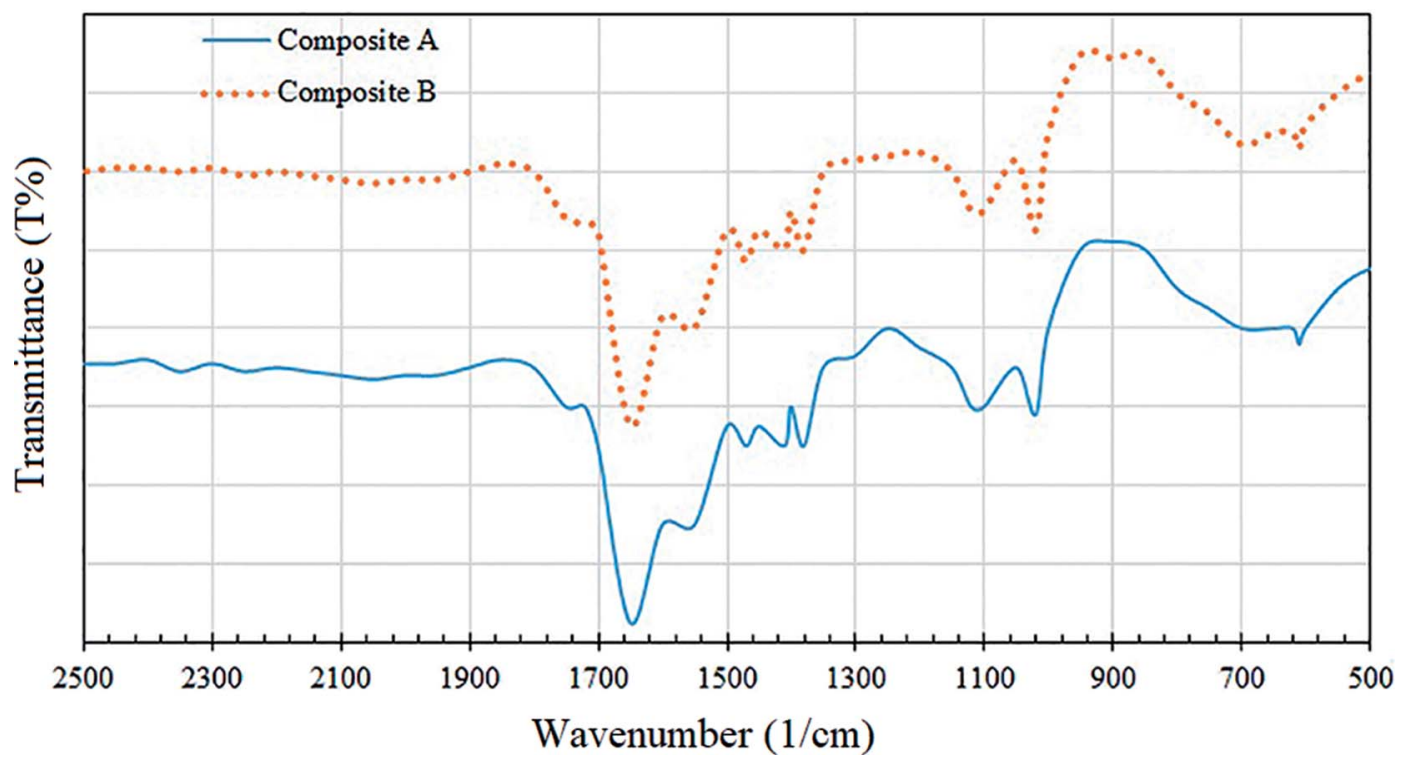

Fig. 9 FTIR spectra of the nanocomposites A and B. 


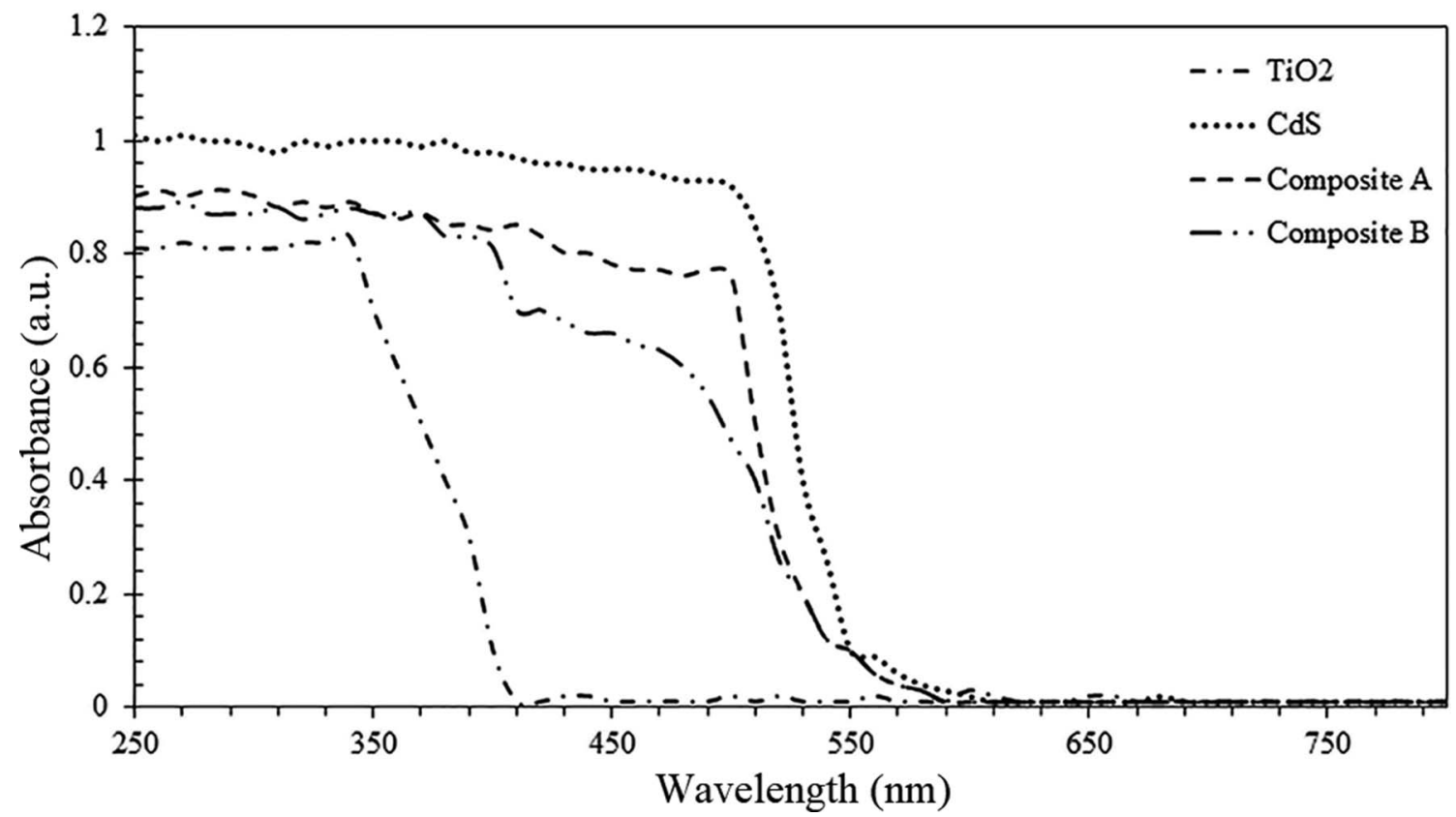

Fig. 10 UV-vis absorption spectra of both composites and pure components.
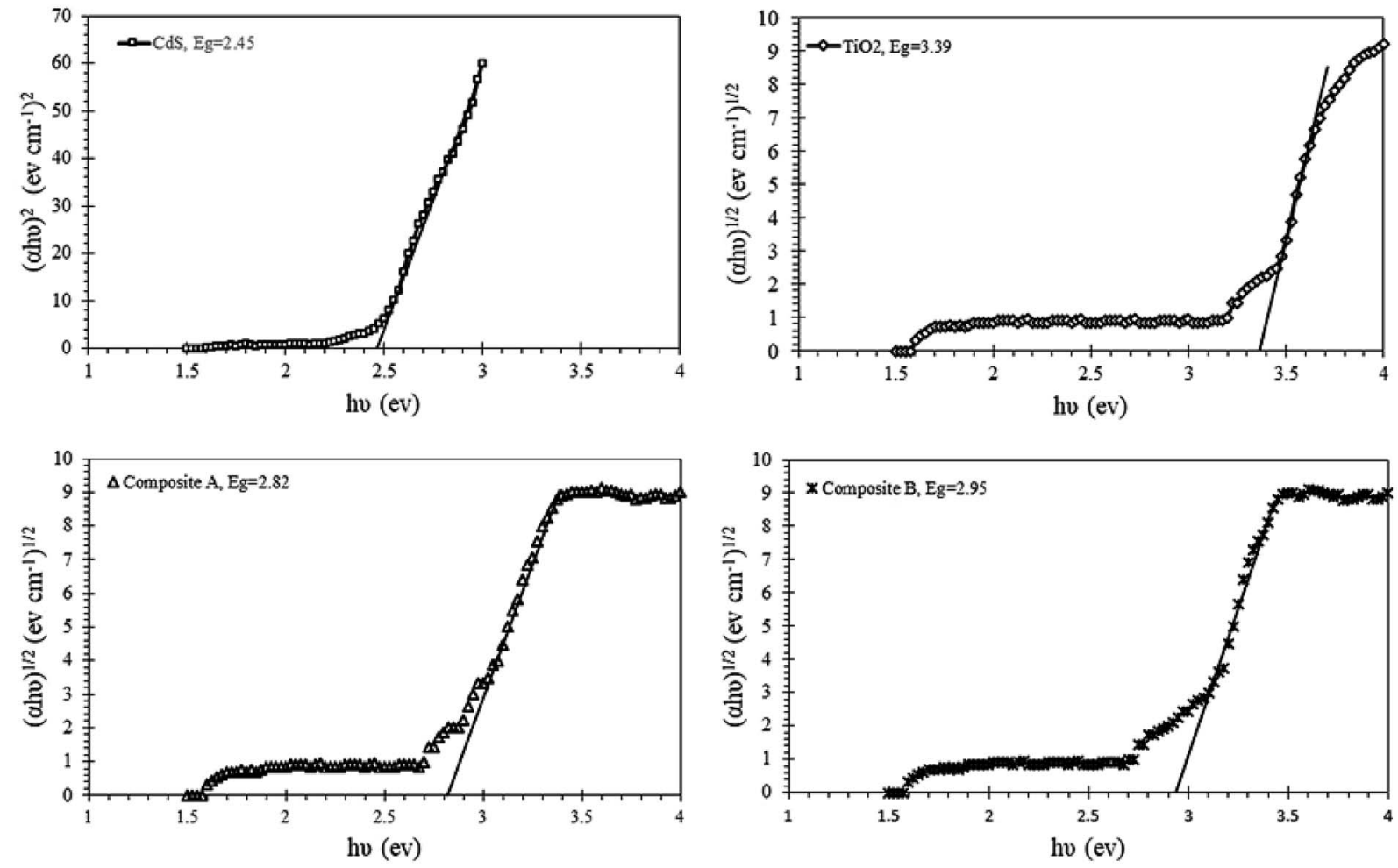

Fig. 11 Band gap of the composites and pure semiconductors.

\subsection{Nucleation and growth mechanism}

The formation of CdS nanoparticles is based on the reaction between ethylenediamine, hydrated cadmium chloride and carbon disulfide present in the aqueous phase and oil phase.
The chemical and physical effects of the ultrasound arise from acoustic cavitation, which is responsible for the mixed phase reactions and mass transfer between the two phases. ${ }^{2,25,36}$ In the absence of surfactants, cavitation plays the same role as microemulsion in mixing the aqueous phase and oil phase. On 
adding cadmium ions to the oil-water interface of the solution, the CdS nanoparticles are formed, and their formation may be attributed to the following sequence of reactions: ${ }^{37}$ the turbulent flow aids ethylenediamine in attacking the $\mathrm{C}-\mathrm{S}$ bond of $\mathrm{CS}_{2}$; upon polymerization of the product from this reaction, $\mathrm{H}_{2} \mathrm{~S}$ gas is produced and reacts with cadmium ions in the oil-water interface of the solution. Eventually, the polymerized molecules produced in the reaction may bridge the oil droplets and water at the $\mathrm{CS}_{2}$-water surface to prevent agglomeration of the droplets. ${ }^{25}$ Eqn (4a) and (b) show the polymerization reaction of the sulfur source $\mathrm{CS}_{2}$ to produce $\mathrm{H}_{2} \mathrm{~S}$ gas for the synthesis of CdS.

$$
\begin{gathered}
\mathrm{C}_{2} \mathrm{H}_{8} \mathrm{~N}_{2}+\mathrm{CS}_{2} \rightarrow \mathrm{C}_{3} \mathrm{H}_{8} \mathrm{~N}_{2} \mathrm{~S}_{2} \\
n\left(\mathrm{C}_{3} \mathrm{H}_{8} \mathrm{~N}_{2} \mathrm{~S}_{2}\right) \rightarrow\left(-\mathrm{HN}-\mathrm{CH}_{2}-\mathrm{CH}_{2}-\mathrm{NH}-\mathrm{SC}\right) n+\mathrm{H}_{2} \mathrm{~S}
\end{gathered}
$$

After synthesis of the core, TTIP was added drop-wise to the mixture in the presence of ultrasound waves to increase the rate of hydrolysis of TTIP and form the $\mathrm{TiO}_{2}$ shell. ${ }^{22}$ During the formation of the shell, cavitation and its physical and chemical effects are responsible for the deposition of a uniform layer of $\mathrm{TiO}_{2}$ on the CdS nanoparticles. The hydrolysis reaction of TTIP is a simple but efficient way to produce the $\mathrm{TiO}_{2}$ particles. Titanium isopropoxide hydrolyses in the presence of water as per the following reaction:

$$
\mathrm{Ti}\left(\mathrm{OC}_{3} \mathrm{H}_{7}\right)_{4}+2 \mathrm{H}_{2} \mathrm{O} \rightarrow \mathrm{TiO}_{2}+4 \mathrm{C}_{3} \mathrm{H}_{7} \mathrm{OH}
$$

The 1-propanol produced herein is miscible in water, and hence, the $\mathrm{TiO}_{2}$ particles are formed. ${ }^{38}$ Cavitation can also remove contaminants from the particle surface; this causes the formation of uniform clusters. ${ }^{39}$ Highly turbulent flow can also drive the nanoparticles towards each other at very high velocity to form the core-shell particles, especially in spherical shape. ${ }^{40}$

\section{Conclusion}

In this study, CdS particles and $\mathrm{CdS} / \mathrm{TiO}_{2}$ core-shell nanocomposite were successfully synthesized under a mild condition through microemulsion and ultrasonic synthesis methods, respectively. To control the thickness of the $\mathrm{TiO}_{2}$ coating, influential parameters that affect the shell were optimized by means of response surface methodology, and the best combination ratio of these parameters was obtained. The optimization process was aimed to minimize the ratio of $\mathrm{TiO}_{2}$ to $\mathrm{CdS}$ to yield a $10 \mathrm{~nm}$ composite, whereas the other factors were changed freely within their operational range. Finally, it was concluded that the power of ultrasound waves had the most influence on the size of the particles. The second most influential parameters were the retention time of synthesis and precursor proportion, whereas temperature was found to have the least impact on particle size. The interaction between sonic waves and synthesis retention time revealed that the retention time must be kept less than 50 minutes to obtain fine particles through this synthesis method. The thickness of the $\mathrm{TiO}_{2}$ shell could be regulated (from $1.4 \mathrm{~nm}$ to $41.4 \mathrm{~nm}$ ) using the proposed model. The sizes of CdS and composite nanoparticles were investigated via DLS and TEM analyses, respectively. TEM images also revealed complete encapsulation of the CdS particles by the $\mathrm{TiO}_{2}$ shell, which was in a spherical shape with acceptable dispersion of particles. Due to coupling with CdS particles, a red shift in the absorption spectra of $\mathrm{CdS} / \mathrm{TiO}_{2}$ was observed via UV-vis analysis as compared to the case of pure $\mathrm{TiO}_{2}$. There are many advantages of applying the present method. First, it is a low-temperature and short-duration method of synthesis. Moreover, the spherical shape of particles, controlled shell depth and acceptable dispersion of particles could be achieved in the absence of surfactants.

\section{Conflicts of interest}

This project has no financial and personal relation with other people and organizations. The corresponding author paid all costs of the project.

\section{References}

1 D. Mangalaraj and D. N. Devi, Recent Trends in Materials Science and Applications, 2017, vol. 189, pp. 9-17.

2 N. Ghows and M. H. Entezari, Ultrason. Sonochem., 2012, 19, 1070-1078.

3 J. Zhang, J. Gao, X. Sun, Z. Peng and J. Diao, Iran. Polym. J., 2007, 16, 39-46.

4 A. Simchi, E. Tamjid, F. Pishbin and A. R. Boccaccini, Nanomedicine, 2011, 7, 22-39.

5 M. Sahooli, S. Sabbaghi and R. Saboori, Mater. Lett., 2012, 81, 169-172.

6 H. H. El-Maghrabi, A. A. Al-Kahlawy, A. A. Nada and T. Zaki, J. Hazard. Mater., 2018, 360, 250-256.

7 A. A. Nada, H. R. Tantawy, M. A. Elsayed, M. Bechelany and M. E. Elmowafy, Solid State Sci., 2018, 78, 116-125.

8 H. H. El-Maghrabi, A. A. Nada, K. R. Diab, A. M. Youssef, A. Hamdy, S. Roualdes and S. A. El-Wahab, J. Photochem. Photobiol., A, 2018, 365, 86-93.

9 A. A. Nada, M. F. Bekheet, S. Roualdes, A. Gurlo and A. Ayral, J. Mol. Liq., 2018, 274, 505-515.

10 M. A. Deyab, A. A. Nada and A. Hamdy, Prog. Org. Coat., 2017, 105, 245-251.

11 Y. Liu, F. Xin, F. Wang, S. Luo and X. Yin, J. Alloys Compd., 2010, 498, 179-184.

12 R. Brahimi, Y. Bessekhouad, A. Bouguelia and M. Trari, Catal. Today, 2007, 112, 62-65.

13 Y. Bessekhouad, D. Robert and J. V. Weber, J. Photochem. Photobiol., A, 2004, 163, 569-580.

14 S. C. Lo, C. F. Lin, C. H. Wub and P. H. Hsieh, J. Hazard. Mater., 2004, 114, 183-190.

15 R. Brahimi, Y. Bessekhouad, A. Bouguelia and M. Trari, J. Photochem. Photobiol., A, 2008, 194, 173-180.

16 J. P. Cheng, R. Ma, D. Shi, F. Liu and X. B. Zhang, Ultrason. Sonochem., 2011, 18, 1038-1042.

17 L. M. Cubillana-Aguilera, M. Franco-Romano, M. L. A. Gil, I. Naranjo-Rodríguez, H.-H. d. Cisneros and J. M. PalaciosSantander, Ultrason. Sonochem., 2011, 18, 789-794. 
18 N. A. Dhas and K. S. Suslick, J. Am. Chem. Soc., 2005, 127, 2368-2369.

19 K. S. Suslick, Sci. Am., 1989, 260, 80-86.

20 K. S. Suslick, Science, 1990, 247, 1439-1445.

21 S. Alizadeh, S. Sabbaghi and M. Soleymani, Int. J. Nano Dimens., 2015, 6, 271-276.

22 S. S. Lee, K. W. Seo, S. H. Yoon, I. W. Shim, K. T. Byun and H. Y. Kwak, Bull. Korean Chem. Soc., 2005, 18, 269-275.

23 H. H. El-Maghrabi, A. Barhoum, A. A. Nada, Y. M. Moustafa, S. M. Seliman, A. M. Youssef and M. Bechelany, J. Photochem. Photobiol., A, 2018, 351, 261-270.

24 L. L. Lapin, Modern Engineering Statistics, Duxbury Press Wadsworth Publishing Company, 1997.

25 N. Ghows and M. H. Entezari, Ultrason. Sonochem., 2011, 18, 269-275.

26 M. Choquette-Labbé, W. A. Shewa, J. A. Lalman and S. R. Shanmugam, Water, 2014, 6, 1785-1806.

27 G. Khairnar, J. Naik and V. Mokale, Bulletin of Faculty of Pharmacy, Cairo University, 2017, vol. 55, pp. 19-29.

28 S. Chowdhury, F. Yusof, M. O. Faruck and N. Sulaimana, Procedia Eng., 2016, 148, 992-999.

29 M. Zarei, A. Niaei, D. Salari and A. Khataee, J. Hazard. Mater., 2010, 173, 544-551.
30 S. S. Mali, S. K. Desai, D. S. Dalavi, C. A. Betty, P. N. Bhosale and P. S. Patil, Photochem. Photobiol. Sci., 2011, 10, 16521658.

31 D. Bersani, P. P. Lottici and X. Ding, Appl. Phys. Lett., 1998, 72, 73-75.

32 T. Martin and H. Schaber, Spectrochim. Acta, Part A, 1982, 38, 655-660s.

33 C. Wang, H. Zhang, J. Zhang, M. Li, H. Sun and B. Yang, J. Phys. Chem. C, 2007, 111, 2465-2469.

34 A. A. Nada, M. Nasr, R. Viter, P. Miele, S. Roualdes and M. Bechelany, J. Phys. Chem. C, 2017, 121, 24669-24677.

35 S. R. Yadav, P. Mishra, R. Mishra, M. Kumar and A. C. Pandey, Ultrason. Sonochem., 2010, 17, 116-122.

36 H. Wang, J. R. Zhang and J. J. Zhu, J. Cryst. Growth, 2002, 246, 161-168.

37 X. Fu, D. Wang, J. Wang, H. Shi and C. Song, Mater. Res. Bull., 2004, 39, 1869-1874.

38 T. Seto, M. Shimada and K. Okuyama, Aerosol Sci. Technol., 1995, 23, 183-200.

39 K. S. Suslick and S. J. Doktycz, The Effects of Ultrasound on Solids, ed. T. J. Mason, JAI Press, New York, 1990, vol. 1, pp. 197-230.

40 D. Radziuk, D. Grigoriev, W. Zhang, D. Su, H. Móhwald and D. Shchukin, J. Phys. Chem. C, 2010, 114, 1835-1843. 